\title{
A CASE OF DVT REVISITED
}

\section{Dina Corbin, MD; Boutros El-Haddad, MD}

\section{Introduction}

- May-Thurner Syndrome is an uncommon condition characterized by the compression of the left common iliac vein by the right common iliac artery against the fifth lumber vertebra.

- Chronic compression results in intimal hyperplasia leading to stenosis and increased incidence of DVT and the risk increases in young women on oral contraceptives.

- The prevalence in patients undergoing evaluation for venous disorders is $2-5 \%$, mostly in women between second and fourth decades.

\section{Presentation}

- A 32 year-old woman on oral contraceptives presented with DVT of the left popliteal vein. Anticoagulation was started and she was discharged on warfarin.

- Days later, while INR was therapeutic. A repeat ultrasound showed DVT extension to the femoral vein. Venogram showed left popliteal, femoral, and left common iliac vein thrombosis.

- Thrombolytic was given. Repeat venogram showed almost complete resolution of the thrombus. She went home on warfarin.

- Two weeks later, and while INR was therapeutic, a Doppler ultrasound showed popliteal DVT. Hypercoagulable workup was negative as well as work up for malignancy. An IVC filter was placed.

- Angioplasty and stenting of the left common iliac vein was done. The patient was kept on low-dose aspirin and discharged on warfarin. The six-month follow-up venogram was negative for DVT.
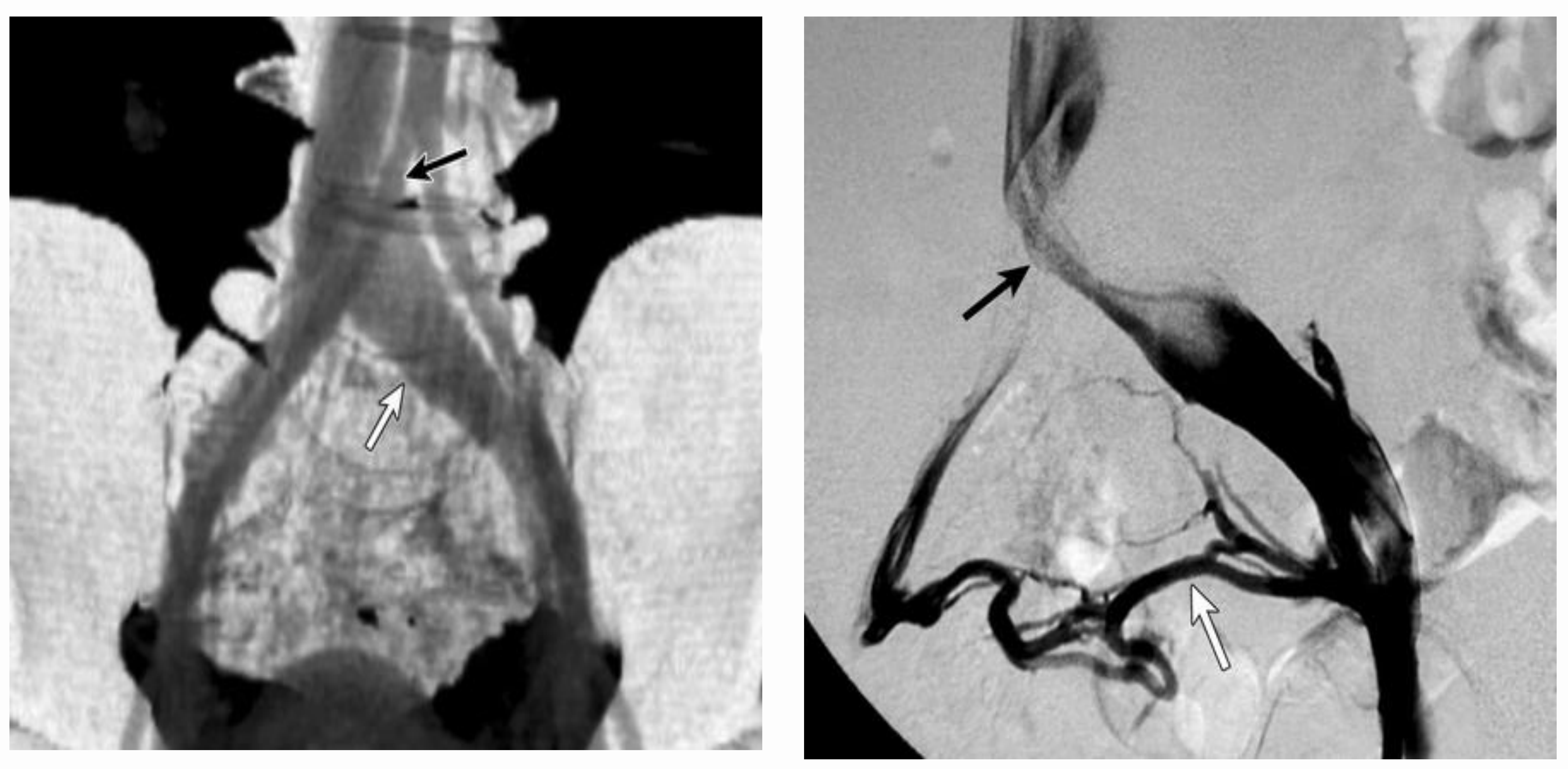

A CT scan (left) and venogram (right) showing compressed left common iliac vein (black arrow).

\section{Discussion}

- Diagnosis of May-Thurner Syndrome is usually made by venography and/or intravenous ultrasound.

- Screening prior to prescribing oral contraceptives is not routinely recommended.

- Anticoagulation therapy alone is not very effective.

- Treatment includes catheter-directed thrombolysis and mechanical thrombectomy with endovascular stent placement.

- We recommend screening in young patients with DVT who failed anticoagulation and have negative hypercoagulable work-up.

\section{References}

1. Murphy EH, et al. Symptomatic ileofemoral DVT after onset of oral contraceptive use in women with previously undiagnosed May-Thurner Syndrome. J Vasc Surg 2009; 49:697-703.

2. Jeon UB, et al. May-Thurner syndrome complicated by acute iliofemoral vein thrombosis: helical CT venography for evaluation of long-term stent patency and changes in the iliac vein. AJR Am J Roentgenol 2010,195:751-757.

3. Loukas M, et al. A case of May-Thurner syndrome. Case report. Folia Morphol 2008; 67(3):214-217. 